\title{
Study of Gender Identity Features in Adolescent Orphan Girls: Russian View
}

\author{
Irina S. Bubnova1, , Larisa V. Tatarinova², Viktoriya I. Rerke ${ }^{3}$, Natalya M. Balabina ${ }^{4}$, Olga V. \\ Zhigalova $^{4}$, Rashad A. Kurbanov ${ }^{5}$, Lidiya S. Cherekhovskaya ${ }^{6}$ \\ ${ }^{1}$ Department of Social Psychology and Management Sociology, Kuban State University, Krasnodar, Russian Federation. \\ ${ }^{2}$ Department of English Philology, Irkutsk State University, Irkutsk, Russian Federation. \\ ${ }^{3}$ Department of Social Pedagogy and Psychology, Irkutsk State University, Irkutsk, Russian Federation. \\ ${ }^{4}$ Department of Policlinic Therapy and General Medical Practice, State Medical University, Irkutsk, Russian Federation. \\ ${ }^{5}$ Department of Civil Law Disciplines, Plekhanov Russian University of Economics, Moscow, Russian Federation. \\ ${ }^{6}$ Research and Educational Laboratory of Remote Access on the Orthodox Journalism, Faculty of Communication Management, Russian State
}

\begin{abstract}
Received: 18/05/2020
Accepted: $30 / 08 / 2020$

Published: 20/09/2020

Abstract

Teenage orphans are experiencing difficulties in awareness of themselves and their place in the world, and the lack of knowledge about the differences of the sexes a vague idea of teenagers-orphans about their future gender roles in the family, the absence of adequate specimens for identification, etc. lead to a breach them of the process of formation of gender identity. The purpose of the article is to study the features of the formation of gender identity in adolescent girls left without parental care, and to develop appropriate recommendations for its formation. The authors studied in detail the features of gender identity in orphans. The features of gender identity in girls in the deprived conditions of the center for children left without parental care are revealed, the value of the type of gender identity for the level of their social adaptation is established. The obtained results allowed to develop practical recommendations on the formation of the type of gender identity in orphans, adequate to their biological sex.
\end{abstract}

Keywords: Gender, Sex, Gender socialization, Sexual socialization, Orphan, Teenager, Masculinity, Femininity, Androgyny, adaptation, Gender identity

\section{Introduction}

The problem of gender socialization of adolescents is of very wide interest among researchers, since it includes questions of the formation of the child's psychological sex, mental gender differences, and gender differentiation as one of the most important and relevant problems of modern psychological science.

According to such researchers as S.L. Bem [1], I.S. Kletsina [2] gender socialization leads to the fact that boys and girls acquire certain qualities that lead to the formation of different types of gender identity. Gender is a sociocultural construct of sex [2], consisting of a set of characteristics and signs of male and female way of thinking and behavior, ideas about norms and rules of behavior, etc. [2]. Thus, sex is the result of evolution, while gender is the result of socialization. Unlike biological sex, which is a set of genetically defined anatomical and physiological characteristics of a person, gender is built in a specific socio-cultural context in a certain historical period and, therefore, is different in time and space. The proper formation of gender identity is influenced by the closest social environment of the child [3]. From psychological and pedagogical research of A.S. Mutadzhirova [4], it is known that for successful sexual role socialization and identification of children and adolescents, a significant adult is needed to guide the formation of a positive "I-concept" of a teenager as a representative of the gender, treat him(her) and communicate with him(her) just like a girl or boy, i.e. would have a differentiated pedagogical effect on the behavior and consciousness of a teenager according to his gender [5]. Thus, the immediate social environment broadcasts to the child specific patterns of behavior, "images-standards" of masculinity or femininity, which they learn and integrate in their behavior, manner and activity. The development of gender identity is determined by family relationships. Intra-family conflicts, the transformation of the system of gender-role behavior play a negative role in this process and can affect the formation of an undifferentiated type of gender identity [6]. Researches of V.S. Mukhina [5], L.N. Shipitsina [7], A.M. Prikhozhan, N.N. Tolstykh and T.I. Yufereva [8], G.N. Semya [9], show that adolescents - pupils and graduates of boarding schools experience difficulties in understanding themselves and their place in the world, show insecurity in understanding their own self. G.N. Semya [9] also indicates the lack of knowledge about the differences between the sexes, the vague idea of orphans about their future sexual role in the family, the difficult formation of gender-role normative orientations.

Summarizing the above, we can conclude that the lack of adequate samples for identification leads to the formation of distorted ideas about masculinity or femininity in orphaned children. Violations of the process of forming gender identity in orphaned teens, as can be seen from the analysis of the literature, are caused by

- The lack of conditions for social trials, improper organization of communication between adults and children, which do not compensate for developmental defects caused by the absence of a family;

Corresponding author: Irina S. Bubnova, PhD in Psychology, Associate Professor Department of Social Psychology and Management Sociology, Kuban State University, Krasnodar, Russian Federation. E-mail: irinaz-bubnova@yandex.ru 
- The lack of psychological and pedagogical preparedness of educators in the formation of adequate sexual identification of pupils of different ages;

- Mainly "female" education, frequent change of adults in the institution, deprivation and the constant presence of children in a collective environment;

- Lack of a positive role for family education $[16,10]$.

\section{Methodology}

\subsection{Degree of Problem development}

Gender identity [2] is an aspect of self-consciousness of the person, which is associated with the experience and description of a person as a representative of a certain sex; it determines the degree to which each individual identifies oneself as a man, woman or a combination of both. Gender identity in the concept of O.A. Voronina [11] is awareness of the personality of its connection with the cultural definitions of masculinity and femininity; in the theory of N.K. Radina and E.Yu. Tereshenkova [12] it is belonging to a particular social group based on gender.

Gender identity, thus, is one of the basic characteristics of personality, which is formed as a result of psychological interiorization of male or female traits, in the process of interaction of "I" and others, in the course of socialization. In this regard, we note that in the epigenetic scheme of E.G. Erickson [13], the basic conflict of the sixth stage of psychosocial development - intimacy versus isolation - is directly related to the gender identity of the individual. At the same time, as shown in a number of works by V.A. Ilyin [14, 15], the results of the resolution of this conflict are not confined to the sphere of intimate-personal relations but are manifested in a specific way in a much broader context of interpersonal relations [16]. However, the ability of the individual to establish full-fledged close relationships in any case does not mean the formation of a fixed positive attitude towards the social environment, but rather is the ability to wide variability in the continuum "acceptance - rejection" while maintaining the integrity of the personality.

Summarizing the above, we emphasize that gender identity acts as a product of social construction and, at the same time, as one of the key factors mediating behavioral activity and personality attitudes in the context of interpersonal relationships [2].

In the structure of gender I.S. Kletsina [17] and S.L. Bem [1] identified the following components: cognitive or gender identity ("I know that I am a man / woman"); emotional or gender identity ("I feel like a man / woman"); behavioral or gender roles and specifics of behavior ("I behave like a man / woman").

S.L. Bem [1] identified and justified in her research 3 types of gender identity:

- Masculine - expression of preference for instrumental styles of activity, energy, assertiveness, the ability to significant, but short-term effort;

- Feminine - commitment to activities related to communication, perception of nuances, subtlety of feelings, the ability to maintain activity that does not require significant effort for a long time;

- Androgynous - the manifestation of masculine and feminine traits at the same time.

Sexual identification, deeply penetrating into the selfconsciousness of the individual, sets the vector of socialization of the individual. For successful adaptation and integration into society, as a full member, the child must be brought up in a family where the formation of gender-specific normative orientations and behavioral patterns occurs by imitation of representatives of their gender, identification with them, as well as in the process of separation from the opposite sex at certain age periods [5]. However, children brought up in boarding schools have limited opportunities for learning these orientations. The problem of social orphanhood is becoming catastrophic every year, being one of the negative trends in modern society. So, the annual increase in the number of orphans is due to the unfavorable socio-economic situation in the country, the decline in moral standards in society $[18,19]$, the degradation of the institution of the family and family values, the increase in the number of families in a socially dangerous situation, narcotization and alcoholization of the population, etc. At present the overwhelming majority of pupils of centers for assistance to children without parental care are social orphans [9].

The problem of working with orphans was considered in the Russian psychology in the works of V.S. Mukhina [5], A.M. Prikhozhan and N.N. Tolstykh [20], I.S. Bubnova and A.G. Tereshchenko [19], V.I. Rerke [21] and others. Emphasizing the fruitfulness of these studies, it should be recognized that outside the field of view of scientists are aspects of the formation of sexual identification of children brought up in orphanages [22-25]. Based on the research of E.S. Morozova [26], we note that the category of children and adolescents brought up in boarding schools is characterized by the development of an imaginary or otherwise blurred (distorted) identity, which is due to maternal - mental and social deprivation [27 - 29].

In both real and social orphans, imaginary identity occurs as a result of a breakdown in emotional attachments between mother and child. The child replaces the missing object for identification with diversified indefinite external reality, it is mystified, arises due to the illusion and the inability to test reality adequately. Thus, a diffuse understanding of oneself is created, identification with a "blurred mother", because the image of the mother in this case is replaced by interaction with a group of children from an orphanage or boarding school, teachers, conditions and the system of a boarding school [3033].

\subsection{Experimental Base and Methods of Research}

This study was conducted during 2018-2019 on the basis of a number of state-owned public institutions of social services "Center for helping children without parental care" in Irkutsk, Ulyanovsk, Irkutsk region and Krasnodar Territory. 250 teenage girls (13-15 years old) took part in the experimental work; the control and experimental groups of these girls were distinguished, based on a diagnostic study. The study of the formation features of the gender identity type in teenage girls left without parental care was carried out using the following techniques: Sex-role questionnaire S.L. Bem [1], Questionnaire "Who Am I?" M. Kuhn and T. McPartland [34], Technique for the diagnosis of socio-psychological adaptation C.R. Rogers and F.D. Daimond [35]. Thus, the most effective methods of psychological diagnostics were chosen, which allow to determine the level of socio-psychological adaptation of respondents, determine their type of gender identity, and study the meaningful characteristics of personality identity.

\section{Results}

Diagnosis of the type of gender identity in orphans using the S.L. Bem [1] questionnaire made it possible to state the following results. $50 \%$ of the respondents in the control group and $70 \%$ of the respondents in the experimental group were diagnosed with androgynous type of gender identity. As noted above, such a person can combine and manifest significant 
features of both masculine and feminine types, but at this stage of ontogenesis, they constitute a risk group, because, taking into consideration the conditions of the Center for assistance to children left without parental care, we can conclude that the gender identity of adolescents is blurred, not formed. Figure 1 shows that in the control group, $10 \%$ of girls have a masculine type of gender. In the experimental group, $30 \%$ of subjects with a masculine type of gender were identified. The results obtained indicate that for a girl they demonstrate such characteristics that are typical to male ones: independence, dominance, assertiveness, risk appetite, independence, aggressiveness, selfconfidence, etc. The obtained results indicate that a gender type that is adequate to the biological gender is not accepted. $40 \%$ of the respondents in the control group revealed a feminine type of gender. For girls of this group the following features are characteristic: passivity, responsiveness, gentleness, preoccupation with motherhood, caring, emotionality. In the experimental group of girls studied, a feminine type of gender identity was not identified. The results obtained indicate that a group of girls with identified masculine and androgynous types of gender identity need specially organized psychological and pedagogical work aimed at adopting a gender type that is adequate to their biological gender. In order to identify gender roles in the structure of the "I-concept" of the personality, as well as to study the meaningful characteristics of gender identity, we used the questionnaire "Who am I?" [34].

Carrying out this technique allowed us to formulate the following conclusions. In the control group, only $17 \%$ of the girls examined chose the right family and interpersonal roles, for example, such as: "I am the future mother, daughter, sister, classmate." Formed professional roles were identified in $22 \%$ of the surveyed girls in the control group. Teenagers indicated such answers as: "I am a future veterinarian, artist, teacher." Other social roles belonged to $10 \%$ of respondents.

Summarizing the results, we found that in the control group, feminine type of gender identity was detected in $17 \%$ of subjects, masculine type of gender identity in $4 \%$, neutral characteristics were inherent in $30 \%$ of subjects in the control group. In the experimental group of teenage girls, we found that only $12 \%$ of the girls surveyed had family roles. $27 \%$ of girls identified professional roles ("I am a future dancer, firefighter, programmer, student").

Thus, in the experimental group of the studied adolescents, we revealed a feminine type of gender identity in $5 \%$ of the adolescents surveyed, a masculine type in $20 \%$ of the examined girls, and neutral characteristics of gender identity are in $36 \%$ of the respondents. Comparing the results of the diagnostic methods, we can draw the following conclusion: the androgynous type of gender identity predominates among the interviewed orphans of adolescence (70\%). The revealed masculine type of gender identity in adolescents (30\%) indicates a problem of inadequately gender-formed identity, which is characterized by an external manifestation of feminine patterns of behavior, and by an internal emotional dissatisfaction with one's biological gender, gender role conflict.

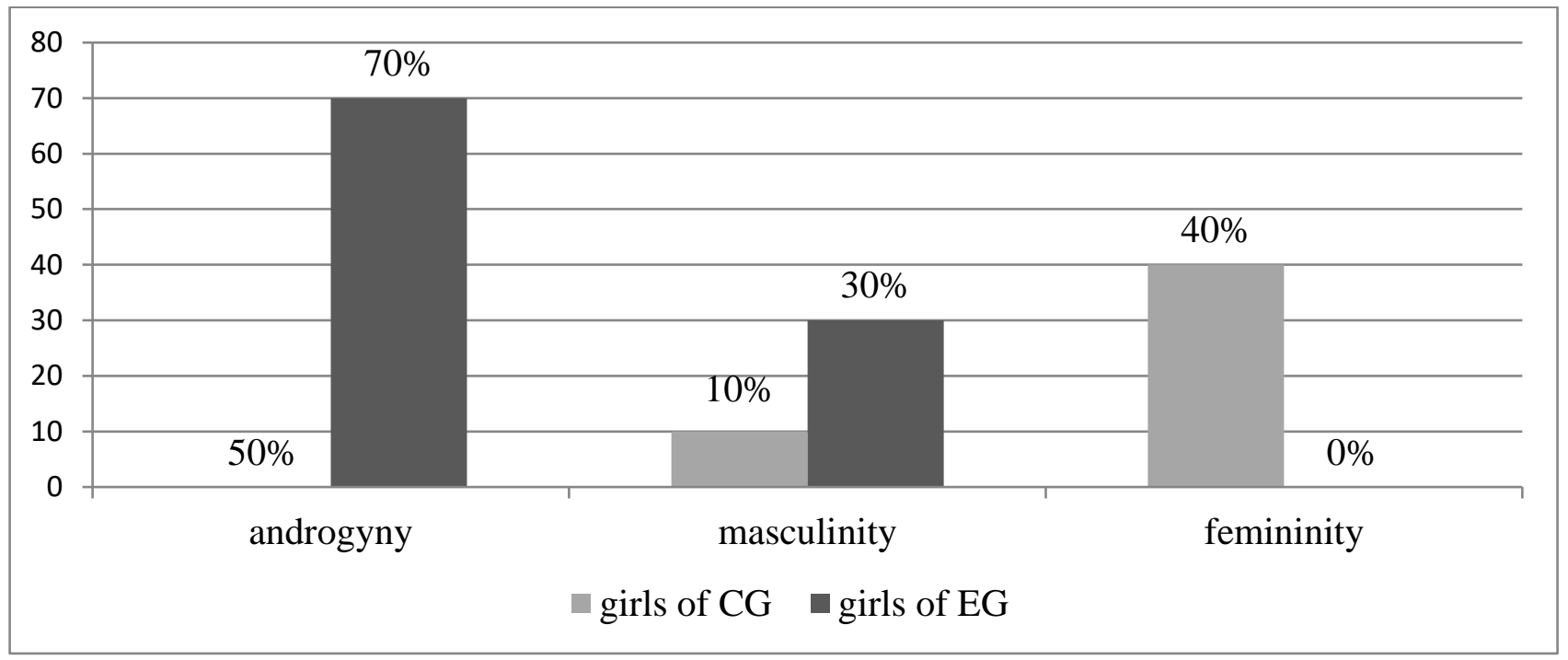

Figure 1: Data obtained during the study of the gender identity type of adolescent girls ("Sex-role questionnaire" S.L. Bem [1]), \%

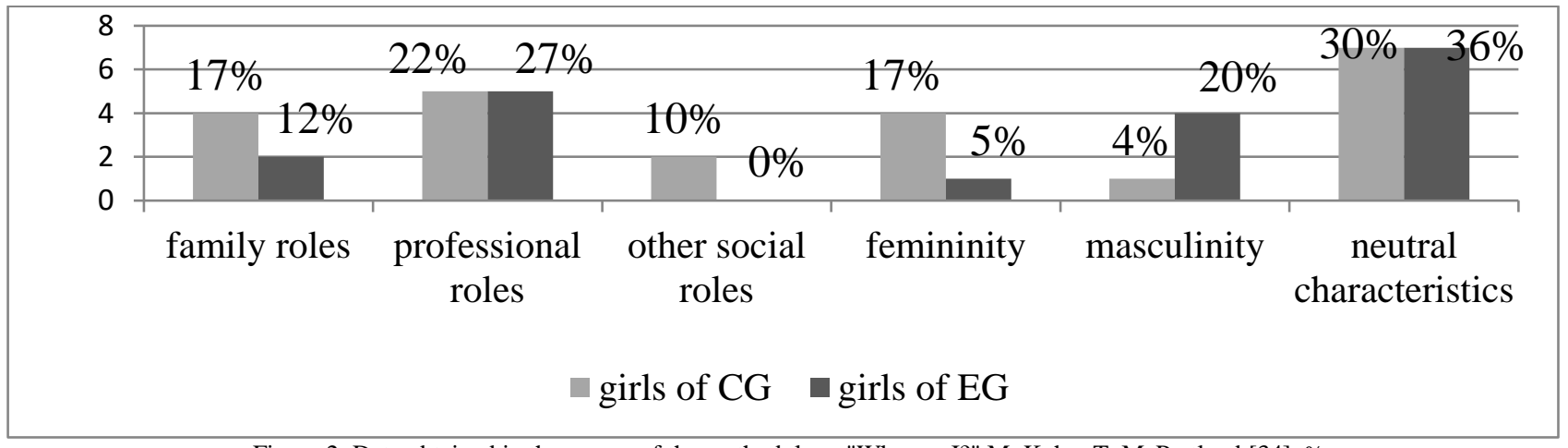

Figure 2: Data obtained in the course of the methodology "Who am I?" M. Kuhn, T. McPartland [34], \% 
Thus, in adolescent girls with a feminine type of gender identity, there is a commitment to those activities that are associated with communication, perception of nuances, subtlety of feelings, the ability to maintain activity that does not require significant effort for a long time. Such qualities as care, modesty, warm relationships with others, empathy are also noted. Adolescent girls with a gender identity not adequate to their biological sex (masculine and/or androgynous) are characterized by rudeness, rivalry with boys, in communication, as a rule, they prefer male companies, avoid caresses in relationships with female educators [36-39].

In order to identify the characteristics of the adaptation period of the personality through the integral indicators "adaptation", "self-acceptance", "acceptance of others", "emotional comfort", "internality", "desire for dominance", we used the technique for diagnosing the socio-psychological adaptation of C.E. Rogers and F.D. Diamond [35]. Carrying out this technique showed that the level of adaptation in the two groups of girls studied is at a low level, since the indicators of the methodology scales are at the level of values less than 65 points. The low level of adaptation is characterized by the fact that adolescents are dominated by a depressed mood, there is a violation of discipline, inattention and aggressiveness are manifested. The level of self-acceptance in the control and experimental group of teenage girls is at an average level, which allows us to conclude about a positive self-esteem of their personal qualities, self-satisfaction, self-confidence.

The criterion of "acceptance of others" in both groups is also at an average level (20 points), which indicates a moderate tolerance for other people, their weaknesses and disadvantages; adolescents are critical of others, tend to give both positive and negative assessments of their personality and behavior. The level of emotional comfort in the control group (14 points) is lower by 2 points than in the experimental group (16 points), which indicates anxiety, tension, pessimism, emotional imbalance. The criterion of "internality" is characterized by the fact that adolescents are dominated by internal motivation over external, they are demanding of themselves, self-critical, tend to rely on their own strength, not counting on someone's help. If to compare the two groups, it should be noted that the level of internality in the experimental group is less by 4 points than in the control group. The criterion of "dominance" is a moderate desire to influence others, to convince them of their opinion, to defend their positions; in the control group it is at an average level, in the experimental group it is at a low level. Analyzing the results obtained in the course of the study, we can conclude that adolescents are at different levels of adaptation: high, medium (risk group) and low (social maladjustment).

A high level of adaptation includes $20 \%$ of the studied adolescents (feminine girls). The study also showed that this category of adolescents has an adequate gender type of their own gender identity, the professional roles of these adolescents are also adequate to the feminine type of gender identity. $45 \%$ of adolescents are at the average level of adaptation. This level is for adolescents "risk group" for maladjustment. Androgynous girls whose social roles are distorted or not formed are at this level of adaptation. The research showed that $35 \%$ of adolescents surveyed are at a low level of adaptation. Their gender identity type is not adequately formed, professional roles are also inadequate to their gender identity type. Therefore, masculine girls differ in that they are aggressive, prone to risk, assertive, tend to dominate and be independent. In conclusion, we note that the results of the current experiment convincingly demonstrated the need to organize a program aimed at preventing the social maladaptation of teenage girls left without parental care, through the formation of a sexually appropriate type of their own gender identity. We identified the tasks of the psychological and pedagogical program of prevention of social maladjustment of adolescents left without parental care, taking into account the type of gender identity, which were successfully solved in the course of its implementation. Therefore, control carrying out of techniques showed: increase of indicators of adaptation at girls-orphans in experimental group, increase of indicators of "self-acceptance" and "acceptance of others"; teenagers began to experience fewer difficulties at interaction with the nearest environment. Girls left without parental care with an inadequately sex-formed type of gender identity, have a tendency to form a gender identity that is adequate to their biological sex.

In conclusion, we note that the results of a stating and control experiment convincingly demonstrated the need for organizing socio-pedagogical preventive work with diagnosed adolescents, as well as developing a preventive program taking into account gender differences.

\section{Practical Recommendations}

The obtained results made it possible to develop practical recommendations aimed at the formation of a sexually appropriate type of their own gender identity as a means of preventing maladjustment in adolescents left without parental care. For this purpose, it was recommended to carry out activities aimed at: the formation of ideas about the basic concepts of gender culture (gender, gender culture, gender roles, gender stereotypes, etc.); the formation of ideas about gender-appropriate types of professional activity; increasing the adaptive capacity of the individual. Thus, classes were held on the topics: "the Duties of men and women in modern society", "the Ideal image of men and women", "If I became a man/woman for a day", "Youth at risk", etc. As a result of the classes for orphans, the following will be formed: a) an adequate idea of their own gender, corresponding to their biological gender; b) about gender stereotypes in society;

- About gender relations and the role of men and women in society; and c) about demonstration and personification of masculinity and femininity in adolescence.

Skills of cooperation and coexistence with people who have the opposite view or opinion on one problem are supposed to be developed as well as the interest in gender-adequate activities.

\section{Discussions and Conclusion}

A theoretical analysis of the research problem led to the conclusion that the process of forming gender identity in adolescents - social orphans can be considered from the point of view of the gender identity crisis. The phenomenon of the crisis of gender identity reflects a situation in which models of masculine and feminine behavior, represented by carriers of male and female identities, turn out to be largely inconsistent with normative models of true masculinity and femininity. This phenomenon is no longer psychological, but social psychological and social in nature and should be analyzed taking into account the influence of various factors. That is why the cause of confused identity is the social status of these children [2]. If a teenage girl finds herself in a situation of inability to maintain a standard of generally accepted feminine behavior for a long time, as occurs in the case of orphans, this provokes the appearance of such a feature that can be qualified as a crisis of identity or lack of formation of gender identity; the notions of masculinity and femininity in children of boarding school, as in a mirror, reflect the key to the normal formation of gender identity - the lack of adequate samples for identification $[23,40]$. 
Gender identity in adolescents develops against the background of the formation of one of the main psychological neoplasms of this age - self-consciousness, because only through the accumulation of ideas about himself, their generalization, integration, interiorization, the teenager comes to realize oneself in the unity of all manifestations.

The study found that most of the interviewed orphans are at a low level of adaptation. The study also showed that these adolescents are characterized by an inadequately formed type of their own gender identity: masculinity. The average level of adaptation in adolescents without parental care was found in $45 \%$ of the respondents who differ in unformed (blurred) gender identity.

We developed a program that is aimed at preventing difficulties in interacting with the environment, preventing inadequately formed type of gender identity in adolescents to create a sexually appropriate type of own gender identity as a means of preventing social maladaptation in adolescents without parental care.

Revealing the issues of the formation of gender identity in orphans, it was found that scientific research in the field of studying gender identity in adolescent girls in the scientific literature is not enough. It was found that the concept of "gender identity" is the recognition of one's male or female gender, the person's experience of being a representative of the sex, as a carrier of specific sex-specific characteristics and behavioral characteristics, correlated with ideas about masculinity / femininity.

The indicators of low, medium and high levels of social maladjustment in adolescents left without care, which are determined by masculine, androgynous and feminine type of gender identity, were revealed based on the results of the study.

The study showed that adolescent girls left without parental care, prone to social maladaptation, have an inadequate gender type of gender identity.

In conclusion, we note that the results of the study convincingly demonstrated the need for the organization of socio-pedagogical preventive work with diagnosed adolescents, as well as the development of a preventive program taking into account gender differences.

\section{Ethical issue}

Authors are aware of, and comply with, best practice in publication ethics specifically with regard to authorship (avoidance of guest authorship), dual submission, manipulation of figures, competing interests and compliance with policies on research ethics. Authors adhere to publication requirements that submitted work is original and has not been published elsewhere in any language.

\section{Competing interests}

The authors declare that there is no conflict of interest that would prejudice the impartiality of this scientific work.

\section{Authors' contribution}

All authors of this study have a complete contribution for data collection, data analyses and manuscript writing.

\section{References}

[1] Bem SL. The Lenses of gender. Transformation of views on gender inequality. Moscow: ROSSPEN.2004.

[2] Kletsina IS. Psychology of gender relations: Theory and Practice. St.Petersburg: Alethea.2004

[3] Babitskaya LA, Fedosova IV. Theoretical and empirical understanding of the concept "life perspective of the personality of orphans".Social Sciences.2016;11(27):6451.
[4] Mutadzhirova AS. The problem of gender-role socialization of orphans in the conditions of an institution of boarding type. International scientific research journal: successes of modern science.2016;7(11):127-131.

[5] Mukhina VS. Deprived of parental care: anthology: Studies. manual for students PED. UN-tov and in-tov. Moscow: Enlightenment.1991.

[6] Rymarev NY. Personality characteristics of adolescents with different gender identity: PhD Thesis. Krasnodar.2006

[7] Shipitsina LN. The psychology of orphaned children: a Training manual. St. Petersburg: Publishing house S.-Peter. UNTA.2005.

[8] Prikhozhan AM, Tolstykh NN, Yufereva TI. Adolescence. Mental development of pupils of the orphanage. Moscow: TTS Sfera pri uchastii Yurayt -M.1991.

[9] Semya GN. Russian experience with graduates of boarding schools. 2007.Almanac "Captivity";5:153-165.

[10] Bobkova TS. Gender features of sexual identification of orphans. Branch of Samara state University of Economics: Elektr. Journal.2013;1:100-111.

[11] Voronina OA. Glossary on gender education. Gender studies. A regional anthology of studies from eight countries of the CIS Moscow.2006:443-511.

[12] Radina N.K., Tereshenkova E.Yu. Age and sociocultural aspects of gender socialization of adolescents. Psychology issues. 2006;5:49-50.

[13] Erickson E.G. Identity: youth and crisis. Moscow: Progress. 1996

[14] Ilyin VA. Comparative study of features of psychosocial development of high school students and first-year students of higher educational institutions. Psychological science and education.2009;1:14-22.

[15] Ilyin VA, Sipyagin DV. Possibilities and prospects of using psychosocial approach to the problem of development in the conditions of secondary school. Psychological science and education.2013;20(2):25-33

[16] Stepanova LG. Formation of gender identity in modern boys and girls. Psychological science and education.2009;14(5):67-72.

[17] Kletsina IS. Gender socialization. St.Petersburg: Ed. RGPU im. A. I. Herzen. 1998

[18] Tatarinova LV, Rerke VI, Bubnova IS. Innovative activity of teachers: study and directions of development. Espacios 2019 ; 40(33):6-16.

[19] Bubnova IS, Tereshchenko AG. Features of gender identity in adolescents of deviant behavior. Kazan pedagogical journal.2017; 2(121):166-169.

[20] Prikhozhan AM, Tolstykh NN. Psychology of orphanhood: studies. benefit. St.Petersburg: Peter. 2005.

[21] Rerke VI. Studying the life perspectives of the personality of pupils of the boarding institution in the context of a subjective picture of the world.Vector of science of the tolyattin state university. series: Pedagogy, Psychology.2015;2:174-176.

[22] Bubnova IS, Rerke VI. Formation of gender identity in adolescents-orphans. Bulletin of Irkutsk state technical University.2014;84(1):175-182.

[23] Bubnova IS. Gender display of an orphan teenager.Kazan pedagogical journal.2016;2(115):389-392.

[24] Agadzhanova ER, Salakhova VB. Values and meanings in the context of modes of human existence: to have or to be. Simbirsk Scientific Journal Vestnik.2018; 3(33):12-16.

[25] Kalinina N.V. Social and personal resources to overcome difficult life situations. Simbirsk Scientific Journal Vestnik.2011;1(3):96-101.

[26] Morozova ES. Aggressiveness and personal identity in adolescents-boarding school students: $\mathrm{PhD}$ Thesis Moscow.2016.

[27] Goloshumova GS, Albakova ZA-M, Marchev KV, Kidinov AV, Gustova EA, Salakhova VB, Krasheninnikova NA. The interrelation of environmental and social factors and man's mental health. Ekoloji.2019;28(107):6013-6016

[28] Lekareva EE, Zaretskiy VV, Artamonova EG, Salakhova VB, Efimova OI, Kalinina NV. Comprehensive rehabilitation of minors with deviant and delinquent behavior: The experience of the Russian system of education. Eurasian Journal of Analytical Chemistry.2018;13(1b): em84. 
[29] Romanova AV, Salakhova VB, Ganova TV, Nalichaeva SA, Nazarova LS, Dolzhenko AI. Hardiness as a component for sustainable development of a person's personality: Ecological and psychological aspect. EurAsian Journal of BioSciences.2019;13(2):1833-1840.

[30] Bulychev II. On genderistics and its key concepts. Theoretical journal "CREDONEW".2005;3:26-51.

[31] Ivanova MV, Kovalenko OV. Psychological features of gender identity formation in primary school age. Irkuts:IGPU.2005.

[32] Semina NE. Gender identification as a psychological mechanism of behavior regulation: on the example of adolescents: abstract. Of PhD. Khabarovsk.2003.

[33] Meshcheryakova MI. Features of formation of gender identity of the teenager in incomplete families. Psychologists: electron. journal.2015;1:42-54.

[34] Kuhn M, McPartland. An empirical investigation of selfattitudes. Am. Sociological Review.1954;19(1):68-76.

[35] Rogers CR, and Rosalind FD. Psychotherapy and Personality Change: Coordinated Research Studies in the Client-Centered Approach. Chicago: University of Chicago Press. 1954.

[36] Bocharev VV. Anthropology of age. St.Petersburf: Peter.2001.

[37] Dubovskaya EM, Mishina KS. The Peculiarities of gender socialization in single-mother families. Psychological research.2015; 8(42):9-18

[38] Voronina OA. Textbook on the course "Fundamentals of gender studies". Moscow: MCGI/MHSCES.2001.

[39] Bern, S. Gender psychology. Moscow: Prime-Euroznak.2004.

[40] Kurochkina IA. Development of gender identity of teenagers of institutions of state education. Fundamental researches.2014; 6(9):1357-1363. 